\title{
The Oxidative Stabilities of Polyunsaturated Fatty Acids in Salmon Egg Phosphatidylcholine Liposomes
}

\author{
Eiichi Nara, ${ }^{* 1}$ Kazuo Miyashita, ${ }^{* 1}$ Toru Ota, ${ }^{* 1}$ and Yoshitaka Nadachi ${ }^{* 2}$ \\ ${ }^{*}$ Department of Chemistry, Faculty of Fisheries, Hokkaido University, \\ 3-1-1 Minato, Hakodate 041-0821, Japan \\ *2Janiftec Inc., 1-2-9, Irifune, Chuo, Tokyo 104-0042, Japan
}

(Received June 12, 1997)

\begin{abstract}
Comparative studies on fatty acid composition and oxidative stability indicate the advantages of salmon egg phosphatidylcholines (PCs) for liposomal materials with high contents of n-3 polyunsaturated fatty acids and relatively high oxidative stability. The oxidative stability of salmon egg PC liposomes increased by the addition of cholesterol, decetyl phosphate, and stearylamine. The oxidation of salmon egg PC liposomes was also inhibited by chicken egg albumin and $\alpha$-tocopherol, however, the high concentration of $\alpha$-tocopherol exhibited a prooxidant activity. When DHA-enriched triacylglycerols (TGs) were encapsulated in liposomes of soybean PC, chicken egg PC, and salmon egg PC, respectively, the highest oxidative stabilities of both TG and PC were shown in salmon egg PC liposomes. This result suggests that salmon egg PC is a potentially useful liposomal material for encapsulation of readily oxidizable fish oils.
\end{abstract}

Key words: PC liposome, oxidative stability, salmon egg, encapsulation of DHA

Liposomes have been extensively investigated and developed in the biomedical field as drug delivery systems. ${ }^{1,2)}$ Since they are well characterized, easily made, highly versatile in their carrier properties, and composed of foodacceptable ingredients, their applications in the food industry have been also studied. ${ }^{3-6)}$ Liposomes are vesicles composed of single or multiple phospholipid bilayers that form spontaneously when these lipids are exposed to an aqueous environment. To avoid oxidative destruction of liposomes, saturated phospholipids have been used for the production of liposomes, however, polyunsaturated phospholipids are an attractive material for the development of liposome-based pharmaceuticals and food materials, because they have characteristic biological functions and physicochemical properties.

Salmon egg contained large quantities of phospholipids, and their major polyunsaturated fatty acids (PUFAs) were 22:6n-3 (DHA), 20:5n-3 (IPA), and 22:5n-3. ) The n-3 PUFAs contained in salmon egg phospholipids are known to be of physiological importance, and the liposomes prepared from them are expected to show some remarkable physicochemical features such as low phase-transition temperature and high fluidity. In order to investigate the potential of salmon egg PC for the liposomal materials, this paper describes the oxidative stability of salmon egg PC liposomes, inhibition effects of some food components on the liposome oxidation, and the oxidative stability of DHA-enriched triglycerides in the liposomes.

\section{Materials and Methods}

\section{PC Preparations}

Soybean PC and salmon egg PC were prepared from commercial soybean lecithin and salmon egg as described previously. ${ }^{8}$ Chicken egg PC was obtained by successive purification with silicic acid column chromatography, preparative thin-layer chromatography (TLC), and high performance liquid chromatography (HPLC) from egg yolk lecithin, which was kindly donated by Q.P. Co., Tokyo, Japan. Each PC gave only a single spot (Rf 0.54 ) by TLC using Silicagel 60 plates (Whatman Paper Ltd., NJ, USA) and chloroform/methanol/water (25:20:2, $\mathrm{v} / \mathrm{v})$ as the solvent system. Neither PC contained tocopherols and peroxides, as determined by HPLC., ${ }^{9,10)}$ The fatty acid composition of each PC was analyzed by capillary gas chromatography (GC) after converting the fatty acyl components in the PC to their methyl esters. GC analysis was performed as described previously."

\section{Preparation and Oxidation of PC Liposomes}

Chicken egg albumin (minimum: $98 \%$ by agarose electrophoresis) was purchased from Sigma Chemical Co., St. Louis, MO, U.S.A. Cholesterol was purchased from Serdary Research Laboratories Inc., Ont. Canada. D- $\alpha$ tocopherol was purchased from ICN Biomedicals Inc., Ohio, USA. Iron sulfate $\left(\mathrm{FeSO}_{4}\right)$ was purchased from Nacalai Tesque Inc., Kyoto, Japan. L $(+)$-ascorbic acid (AsA), dicetyl phosphate (DCP), and stearylamine (SA) were purchased from Wako Pure Chemical Industries, Osaka, Japan.

Correspondence author: Kazuo Miyashita (Department of Chemistry, Faculty of Fisheries, Hokkaido University, 3-1-1 Minato, Hakodate 041, Japan). 
PC liposomes (small unilamellar vesicles) were prepared in the following manner. PC was dissolved in chloroformmethanol. The solvent was then removed by gently sweeping with nitrogen. A $0.05 \mathrm{M}$ phosphate buffer (pH 7.4 at $37^{\circ} \mathrm{C}$ ) was added to the dried film of PC. The solution was shaken and sonicated on ice for $5 \mathrm{~min}$ with a Sonicator 5202 PZT (Ohtake Works Co., Tokyo, Japan). Cholesterol, $\alpha$-tocopherol, stearylamine, and dicetyl phosphate were added by dissolving them with the chloroformmethanol. Chicken egg albumin was added by mixing it with the phosphate buffer. PC liposome solution $(10 \mathrm{~m} l)$ was pipetted into a flat-bottomed glass tube $(30 \mathrm{ml}, 2.6 \mathrm{~cm}$ i.d.), and oxidation was then initiated by adding $50 \mu \mathrm{l}$ of an $\mathrm{FeSO}_{4}$-AsA aqueous solution. The reaction mixture $(10.05 \mathrm{ml})$ contained $250 \mu \mathrm{M}$ of $\mathrm{PC}, 1.0 \mu \mathrm{M}$ of $\mathrm{FeSO}_{4}$, and $20.0 \mu \mathrm{M}$ of AsA, respectively. Incubation was performed in the dark at $37^{\circ} \mathrm{C}$.

\section{Extraction and GC Analysis}

After a timed period of incubation, the oxidized $P C$ in liposomes was extracted with chloroform/methanol (2:1, $\mathrm{v} / \mathrm{v}$ ) and the decrease in unoxidized polyunsaturated fatty acyl component in PC during oxidation was estimated from the change in the ratio of total peak areas of 14 kinds of major PUFAs to that of stearic acid by GC. The procedures for extraction, transmethylation, and $\mathrm{GC}$ analysis were the same as used previously. ${ }^{8)}$

\section{Encapsulation of DHA-TG in Liposomes}

Modified tuna oil with enhanced DHA contents was kindly donated by Maruha Co., Tsukuba, Japan. This oil was passed through a column packed with a n-hexane slurry of 1:1 mixture $(w / w)$ of activated carbon and Celite 545 for removal of tocopherols. ${ }^{11)}$ Recovered lipids were fractionated on a silicic acid column eluting with diethyl ether/n-hexane solution. TG was eluted with diethyl ether/hexane (10:90, v/v). Purified TG (DHA-TG) gave only a single spot on TLC, contained no tocopherol, and its peroxide value was zero. The fatty acid composition of DHA-TG was determined by capillary GC as described previously. ${ }^{12)}$

DHA-TG and PC were dissolved in chloroform/ methanol with cholesterol, $\alpha$-tocopherol, and stearylamine. The solvent was then removed and the resulting film was suspended by adding a $0.05 \mathrm{M}$ phosphate buffer $(\mathrm{pH}$ 7.4 at $37^{\circ} \mathrm{C}$ ) containing chicken egg albumin. The final concentration of PC, DHA-TG, cholesterol, SA, $\alpha$ tocopherol, and chicken egg albumin in the liposome solution $(10.05 \mathrm{~m} l)$ were $250 \mu \mathrm{M}, 125 \mu \mathrm{M}, 100 \mu \mathrm{M}, 25 \mu \mathrm{M}, 0.25$ $\mu \mathrm{M}$, and $0.02 \mathrm{wt} \%$, respectively. The liposomes encapsulating DHA-TG were oxidized by adding $\mathrm{FeSO}_{4}-\mathrm{AsA}$ aqueous solution at $37^{\circ} \mathrm{C}$ in the dark. The whole lipids extracted with chloroform/methanol from the liposomes at certain time intervals were fractionated by silicic acid column chromatography into $\mathrm{PC}$ and $\mathrm{TG}$ with chloroform and methanol as a solvent. Each lipid was transmethylated and subjected to $G C{ }^{8,12)}$ Oxidative stabilities of $P C$ and DHA-TG in the liposomes were evaluated by the GC analysis of the decrease rate in total PUFAs of each lipid during oxidation.

\section{Results and Discussion}

The fatty acid profiles of soybean PC, chicken egg PC, and salmon egg PC are listed in Table 1. As reported previously, ${ }^{8)}$ major PUFAs in salmon egg PC were 22:6n-3 (DHA), 20:5n-3 (IPA), and 22:5n-3, whereas soybean PC contained $67.6 \mathrm{~mol} \%$ of $18: 2 \mathrm{n}-6$ (LA). On the other hand, chicken egg PC contained LA, 20:4n-6, 22:5n-6, and DHA as main PUFAs. The average numbers of bisalylic positions in the salmon egg PC molecule, in the chicken egg PC molecule, and in the soybean PC molecule were calculated from their fatty acid compositions to be $4.58,0.87$, and 1.59 , respectively. These profiles imply that salmon egg $\mathrm{PC}$ would be oxidized most rapidly.

However, as shown in Fig. 1, salmon egg PC was shown to be stable to oxidation in liposomes, while soybean PC liposome was oxidized most rapidly. Liposomes are vesicles composed of phosopholipid bilayers. The oxidative

Table 1. Main fatty acid composition of soybean PC, chicken egg $\mathrm{PC}$, salmon egg PC, and DHA-TG

\begin{tabular}{lcccc}
\hline $\begin{array}{c}\text { Fatty acid } \\
(\text { mol } \%)\end{array}$ & $\begin{array}{c}\text { Soybean } \\
\text { PC }\end{array}$ & $\begin{array}{c}\text { Chicken egg } \\
\text { PC }\end{array}$ & $\begin{array}{c}\text { Salmon egg } \\
\text { PC }\end{array}$ & DHA-TG \\
\hline $14: 0$ & 0.1 & 0.2 & 1.5 & 2.2 \\
$16: 0$ & 12.8 & 32.3 & 18.0 & 11.8 \\
$18: 0$ & 3.2 & 12.9 & 9.4 & 2.6 \\
$16: 1 \mathrm{n}-7$ & 0.2 & 1.3 & 1.7 & 3.8 \\
$18: 1 \mathrm{n}-7$ & 1.6 & 1.5 & 3.0 & 1.5 \\
$18: 1 \mathrm{n}-9$ & 8.6 & 27.2 & 9.6 & 10.6 \\
$20: 1 \mathrm{n}-9$ & 0.1 & 0.1 & 0.9 & 1.6 \\
$16: 2 \mathrm{n}-4$ & - & 0.2 & 0.5 & 1.1 \\
$18: 2 \mathrm{n}-6$ & 67.6 & 15.6 & 0.5 & 0.7 \\
$18: 3 \mathrm{n}-3$ & 5.9 & 0.1 & 0.2 & 0.3 \\
$20: 4 \mathrm{n}-3$ & - & - & 0.7 & 0.4 \\
$20: 4 \mathrm{n}-6$ & - & 4.9 & 0.9 & 3.1 \\
$20: 5 \mathrm{n}-3$ & - & - & 16.1 & 4.6 \\
$22: 5 \mathrm{n}-3$ & - & - & 7.0 & 2.2 \\
$22: 5 \mathrm{n}-6$ & - & 1.3 & - & 2.6 \\
$22: 6 \mathrm{n}-3$ & - & 1.2 & 25.0 & 46.6 \\
\hline
\end{tabular}

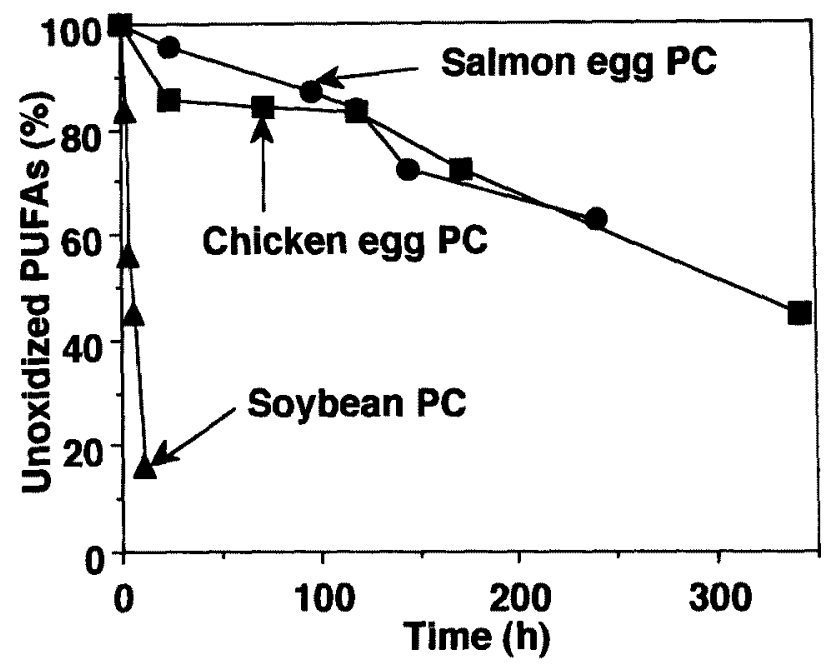

Fig. 1. Comparison of oxidative stability of liposomes prepared from salmon egg $\mathrm{PC}$, chicken egg $\mathrm{PC}$, and soybean $\mathrm{PC}$. 


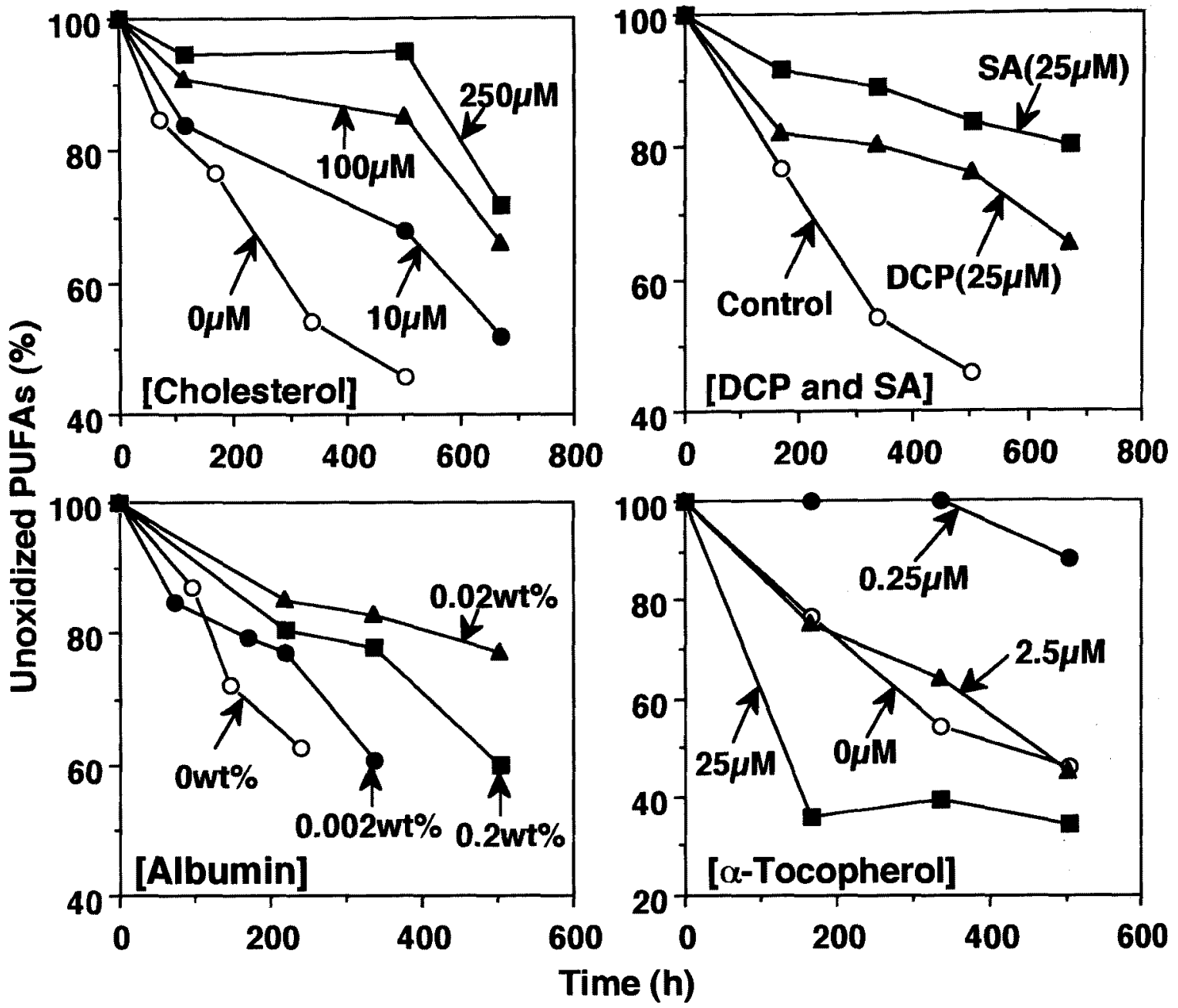

Fig. 2. Effects of cholesterol, DCP, SA, $\alpha$-tocopherol, and albumin on the oxidative stability of salmon egg PC liposomes.

stability of each PC in liposomes is expected to be affected by the conformation of each bilayer. The result in Fig. 1 suggests that it may be difficult for free radicals and/or oxygen to attack PUFAs in the bilayers of salmon egg PC liposomes. In our previous paper, ${ }^{13)}$ we found that the main molecular species of soybean PC was 1,2-dilinoleoyl$P C$, while most of the PUFAs in salmon egg PC and chicken egg PC were not esterified at the sn-1 position but at the sn-2 position. This result suggested that the selective distribution of PUFAs found in salmon egg PC and chicken egg PC may increase the resistance of PC bilayers to oxidation.

Although many papers have been published on the chemical, physiological, and biological properties of liposomes, little is known about the influence of the PUFA composition on the conformation of PC bilayers ${ }^{14)}$ and scant attention has been given to the relationship between the conformational features of PC liposome bilayers and their oxidative stabilities. Therefore, further studies are necessary to investigate the effect of the change of the $\mathrm{PC}$ bilayer conformation on the oxidation of PUFA in PC molecule.

Figure 2 shows the effects of cholesterol, DCP, SA, tocopherol, and albumin on the oxidative stability of salmon egg PC liposomes. The stability increased with increasing molar concentration of cholesterol and by the addition of DCP and SA. Cholesterol incorporated into the PC bilayers has a condensing effect on the bilayer arrangement over phase transition temperature. ${ }^{15)}$ DCP and SA give the negative or positive charge to the liposome, respectively, thus protecting liposome from aggregation. 16,17$)$ Thus, it is suggested that the inhibition of these ingredients on the oxidation of salmon egg PC liposomes would be due to the physiological stabilization of PC liposomes.

Antioxidant effects of chicken egg albumin and $\alpha$ tocopherol depended on their concentrations (Fig. 2). The addition of albumin inhibited the oxidation of salmon egg PC liposomes and the most effective concentration was $0.02 \mathrm{wt} \%$. The strong antioxidant effect of $\alpha$-tocopherol on the liposome oxidation was observed at a concentration of $0.25 \mu \mathrm{M}$, while $\alpha$-tocopherol exhibited a weak antioxidant activity at a concentration of $2.5 \mu \mathrm{M}$ and a prooxidant activity at a concentration of $25 \mu \mathrm{M}$. The antioxidant activity of $\alpha$-tocopherol can be reversed to a prooxidant activity especially when the concentration of $\alpha$-tocopherol increases. ${ }^{18-20)}$ Bazin et al. $^{20)}$ reported that the autoxidation of arachidonic acid (2.5 $\mathrm{mm}$ ) in an aqueous medium was 
Table 2. Changes in the unoxidized PUFAs (\%) during oxidation of PC and DHA-TG in liposomes

\begin{tabular}{lccccc}
\hline \multirow{2}{*}{ Liposomes } & & \multicolumn{4}{c}{ Oxidation time (h) } \\
\cline { 3 - 6 } & Lipid class & 0 & 168 & 336 & 504 \\
\hline Soybean PC & PC & 100 & 73.8 & 12.3 & - \\
\multirow{2}{*}{ Chicken egg PC } & TG & 100 & 36.2 & 26.4 & - \\
& PC & 100 & 97.7 & 56.0 & 46.4 \\
Salmon egg PC & TG & 100 & 53.1 & 10.9 & 6.4 \\
& PC & 100 & 100 & 100 & 75.1 \\
& TG & 100 & 100 & 100 & 30.9 \\
\hline
\end{tabular}

accelerated by the addition of $\alpha$-tocopherol at concentrations of $125 \mu \mathrm{M}$ and $12.5 \mu \mathrm{M}$.

Table 2 shows the oxidative stabilities of liposomal PC and DHA-TG encapsulated in the PC bilayers. The liposomes were prepared from each $\mathrm{PC}$ with $100 \mu \mathrm{M}$ of cholesterol, $25 \mu \mathrm{M}$ of SA, $0.25 \mu \mathrm{M}$ of $\alpha$-tocopherol, and $0.02 \mathrm{wt} \%$ of chicken egg albumin to prevent the PC bilayers from their oxidation. When DHA-TG was encapsulated in PC liposomes, the highest oxidative stability of PC in liposomes was shown by salmon egg PC, followed by chicken egg $\mathrm{PC}$ and soybean $\mathrm{PC}$, respectively, whereas no significant difference was apparent between salmon egg PC and chicken egg PC without encapsulation of DHA-TG (Fig. 1). DHA-TG was also most oxidatively stable when it was encapsulated in salmon egg PC bilayers. Encapsulation of lipophilic substances into a liposome would affect its bilayer nature. ${ }^{21-23)}$ The lower oxidative stability of chicken egg PC than salmon egg PC in liposomes encapsulated DHA-TG may be due to the changes in the packing conformation of PC bilayers by the encapsulation of DHA-TG.

In the preparation and processing of food products, it is desirable to include various components or additives, however, some additives, such as fish oils, are readily oxidized and develop an unpleasant odor and flavor. Haynes et $a$. $^{24)}$ suggested that the encapsulation of these oxidizable unsaturated lipophilic components in saturated liposome bilayers would prevent them from oxidation and could facilitate the dispersion of the lipophilic components in an aqueous phase. The present study shows the possibility that salmon egg PC will also be a useful liposomal material for the prevention of the oxidation of encapsulated fish oils.

The oxidative stability of each lipid was evaluated by monitoring the decrease in PUFAs during oxidation by $\mathrm{GC}$ in the present study. Although GC analysis is useful for comparing the oxidation rate of different types of PUFAs, it gives little information on the lipid oxidation products that are responsible for the development of rancidity in food. The primary products of lipid peroxidation are monohydroperoxides (MHPs), and DHA-MHPs are much more easily decomposed to produce a multitude of secondary oxidation products such as volatile carbonyls. ${ }^{25}$ ) The volatile carbonyls are the most important contributors to rancid and unpleasant flavors. In future studies of salmon egg PC as a liposomal material, it is therefore imperative that the oxidative stability of PUFAs in salmon egg PC liposomes is studied further in respect of the formation of oxidation products, especially of volatile com- pounds, during the early stage of oxidation.

\section{References}

1) T. Sato and J. Sunamoto: Recent aspects in the use of liposomes in biotechnology and medicine. Prog. Lipid Res., 31, 345-372 (1992).

2) B. F. Haumann: Liposomes offer hope as medical tools. INFORM, 6, 793-800 (1995).

3) W. Alkhalaf, J.-C. Piard, M. E. Soda, J.-C. Gripon, M. Desmazeaud, and L. Vassal: Liposomes as proteinase carriers for the accelerated ripening of saint-paulin type cheese. J. Food Sci., $53,1674-1679$ (1988).

4) J. H. Senior, G. Gregoriadis, D. P. R. Muller, Y. V. Pathak, and N. Mclntyre: Liposomes facillitate uptake of lipid-soluble vitamins after oral delivery to normal and bile-duct obstructed rats. Biochem. Soc. Transactions, 17, 121-122 (1989).

5) M. Matsuzaki, F. McCafferty, and M. Karel: The effect of cholesterol content of phospholipid vesicles on the encapsulation and acid resistance of $\beta$-galactosidase from $E$. coli. Int. J. Food Sci. and Tech., 24, 451-460 (1989).

6) C. J. Kirby, C. J. Whittle, N. Rigby, D. T. Coxon, and B. A. Law: Stabilization of ascorbic acid by microencapsulation in liposomes. Int. J. Food Sci. and Tech., 26, 437-449 (1991).

7) T. Ota and T. Takagi: Furan fatty acids of lipids from serum and sexual organs of chum salmon. Nippon Suisan Gakkaishi, 57, 1565-1571 (1991)

8) K. Miyashita, E. Nara, and T. Ota: Comparative study on the oxida tive stability of phosphatidylcholines from salmon egg and soybean in an aqueous solution. Biosci. Biotech. Biochem., 58, 1772-1775 (1994).

9) K. Miyashita and T. Takagi: Tocopherol content of Japanese algae and its seasonal variation. Agric. Biol. Chem., 51, 3115-3118 (1987).

10) C. G. Crawford, R. D. Plattner, D. J. Sessa, and J. J. Rackis: Separation of oxidized and unoxidized molecular species of phosphatidylcholine by high pressure liquid chromatography. Lipids, 15, 91-94 (1980).

11) E. N. Frankel, W. E. Neff, and T. R. Bessler: Analysis of autoxidized fats by gas chromatography-mass spectrometry: $V$. photosensitized oxidation. Lipids, 14, 961-967 (1979).

12) K. Miyashita, M. Hirao, E. Nara, and T. Ota: Oxidative stability of triglycerides from orbital fat of tuna and soybean oil in an emulsion. Fisheries Sci., 61, 273-275 (1995).

13) E. Nara, K. Miyashita, and T. Ota: Oxidative stability of liposomes prepared from soybean PC, chicken egg PC, and salmon egg PC. Biosci. Biotech. Biochem., 61, 1736-1738 (1997).

14) N. Tsubaki, Y. Nakano, Y. Goto, K. Ogino, and M. Abe: Molecular interactions between saturated and unsaturated phospholipids in a monolayer and a bilayer. J. Jpn. Oil Chem. Soc., 41, 551-557 (1992) (in Japanese).

15) J. B. Finean: Interaction between cholesterol and phospholipid in hydrated bilayers. Chem. Phys. Lipids, 54, 147-156 (1990).

16) J. A. Rogers and T. Cheuk: Partial specific volumes of liposome systems. Chem. Phys. Lipids, 53, 211-217 (1990).

17) M. Abe, T. Hiramatsu, H. Uchiyama, H. Yamauchi, and K. Ogino: Molecular interactions between phospholipid and nonionic surfactants in a lipid bilayer. $J$, Jpn. Oil Chem. Soc., 41, 136-141 (1992) (in Japanese).

18) T. P. Labuza: Kinetics of lipid oxidation in foods. CRC Crit. Rev. Food Technol., 2, 355-388 (1971).

19) J. Cillard, P. Cillard, M. Cormier, and L. Girre: $\alpha$-Tocopherol prooxidant effect in aqueous media: increased autoxidation rate of linoleic acid. J. Am. Oil Chem. Soc., 57, 252-255 (1980).

20) B. Bazin, J. Cillard, J.-P. Koskas, and P. Cillard: Arachidonic acid autoxidation in an aqueous media effect of $\alpha$-Tocopherol, cystein and nucleic acids. J. Am. Oil Chem. Soc., 61, 1212-1215 (1984).

21) W. Ehringer, D. Belcher, S. R. Wassall, and W. Stillwell: A comparison of the effects of linolenic (18:3 $\Omega 3)$ and docosahexaenoic (22:683) acids on phospholipid bilayers. Chem. Phys. Lipids, 54, 79-88 (1990).

22) W. D. Ehringer, D. Belcher, S. R. Wassall, and W. Stillwell; A com- 


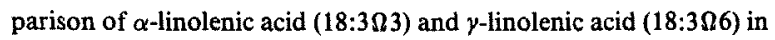
phosphatidylcholine bilayers. Chem. Phys. Lipids, 57, 87-96 (1991).

23) M. Abe, Y. Takao, T. Yamamoto, K. O. Kwon, H. Yamauchi, and K. Ogino: Molecular interactions between phospholipid and lipophilic substances in a lipid bilayer. J. Jpn. Oil Chem. Soc., 41, 404-409 (1992) (in Japanese)

24) L. C. Haynes, H. Levine, and J, W. Finley: Liposome composition for the stabilization of oxidizable substances. U. S. Patent 5015483 (May 14, 1991).

25) S.-Y. Cho, K. Miyashita, T. Miyazawa, K. Fujimoto, and T. Kaneda: Autoxidation of ethyl eicosapentaenoate and docosahexaenoate under light irradiation. Nippon Suisan Gakkaishi, 53, 813-817 (1987). 